\title{
Impact of Multi-wavelength sliceable transponders in Elastic Optical Networks
}

\author{
M. Dallaglio ${ }^{1}$, A. Giorgetti ${ }^{1}$, N. Sambo ${ }^{1}$, L. Velasco $^{2}$, P. Castoldi $^{1}$ \\ ${ }^{1}$ Scuola Superiore Sant'Anna, Pisa, Italy; ${ }^{2}$ Universitat Politècnica de Catalunya (UPC), Barcelona, Spain \\ matteo.dallaglio@sssup.it
}

\begin{abstract}
The utilization of sliceable bandwidth variable transponders based on multi-wavelength technology is evaluated in EONs. A routing and spectrum assignment scheme is proposed specifically designed to consider the constraints introduced by multi-wavelength technology. OCIS codes: (060.0060) Fiber optics and optical communications; (060.4251) Networks, assignment and routing algorithms.
\end{abstract}

\section{Introduction}

Optical transport networks are gradually evolving towards Elastic Optical Network (EON) architecture where the spectrum is exploited by means of a flexible grid where lightpaths occupies a portion of spectrum in multiples of 12.5 $\mathrm{GHz}$ slices. In EONs, it is possible to achieve high spectral efficiency because each lightpath is allowed to use a different amount of spectrum depending on the bit rate and the exploited modulation format [1].

One of the most important upgrade recently introduced to support the EON architecture is the sliceable bandwidth variable transponder (SBVT) [1]. SBVTs are capable to generate multiple optical carriers, which can be merged into high-rate super-channels or routed independently along different paths.

In the traditional SBVT architecture, a dedicated tunable laser is used to generate each carrier, we refer to this SBVT implementation as multi-lasers SBVT (i.e., ML-SBVT). Recent studies introduced a new SBVT architecture using a single multi-wavelength (MW) source able to generate multiple carriers (i.e., MW-SBVT) [2,3]. MW-SBVTs allow an improved super-channel spectrum efficiency because carriers are intrinsically spectrally locked therefore guard bands between carriers can be reduced. In addition, MW-SBVTs are expected to be cheaper by reducing the number of lasers, with lower footprint and lower power consumption [2]. On the other hand, MW-SBVTs introduce new constraints in the routing and spectrum assignment (RSA). Specifically, using a multi-laser source each carrier can be independently tuned in the whole spectrum, whereas a multi-wavelength source imposes a limitation on the maximum spacing among carriers which, in some case, has also to be symmetric [2].

The work in [3] recently presented a testbed based on a programmable multi-wavelength source capable of generating asymmetric channel spacing without the need of additional filters before the modulation block. The work in [4] investigated the utilization of slice-ability applied to the MW-SBVT architecture during provisioning and restoration considering an unlimited number of transponders.

This paper investigates the impact of the utilization of MW-SBVTs compared to the ML-SBVT in EONs with a limited number of transponders per node. Moreover, the paper proposes a multi-wavelength aware RSA scheme (i.e., MWA-RSA) specifically designed to handle the constraints introduced by the multi-wavelength technology.

\section{Routing and spectrum assignment with multi-wavelength transponders in EONs}

Fig. 1 explains the RSA constraints introduced by MW-SBVTs. Assuming an SBVT supporting four carriers with a maximum carrier spacing of $50 \mathrm{GHz}$ (4 slices). Two carriers are already allocated, each one serving a lightpath of three slices (8-10 and 14-16). When a new lightpath request of three slices arrives, the RSA computes the path. According to the available spectrum on the computed path (illustrated in Fig.1), the lightpath could be allocated into slots 1-3, 7-9, 13-15, 19-21 or 20-22. If a ML-SBVT is used, all the slots supported by the path can be allocated except for the slots already allocated by the transponder 7-9, 13-15 (indeed the considered SBVT architecture locally multiplexes all the generated sub-carriers [2]). If a MW-SBVT is used, none of the available slots can be used because the channel spacing constraint limits the allocable spectrum (i.e., MW spacing constraint in Fig. 1).

MWA-RSA scheme: the proposed scheme aims at handle the MW-SBVT constraints during RSA. Specifically, it maps the state of transponders into a virtual graph, which takes into account the constraints related to each specific transponder. In particular, suppose we have the topology illustrated in Fig. 2a, and we need to solve the RSA for a new lightpath between the node pair (S, D). First, a new couple of virtual source/destination nodes is created (Sv, Dv). Then, for each transponder at the receiver and at the transmitter sides, a new virtual node $\left(\mathrm{Tv}_{\mathrm{i}}\right)$ and a couple of virtual directed links are created $\left(\mathrm{Lv}_{\mathrm{i}}\right)$. For each virtual link couple, a spectrum map is computed based on the transponder state and the lightpath request parameters (e.g., number of required slices). After this computation, the spectrum map lists all the possible frequency slots supported by the transponder. At this point, the virtual source/destination nodes 


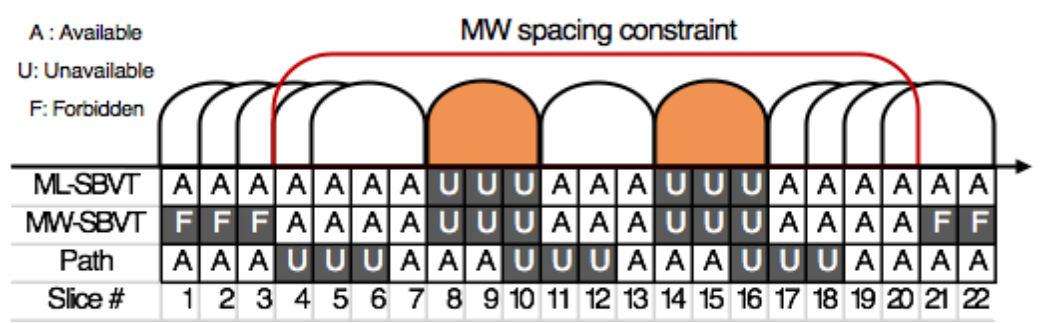

Fig. 1. Multi-wavelength constraint

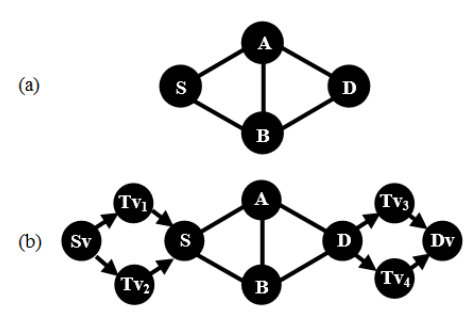

Fig. 2. Real and virtual graphs example

are connected to the corresponding real source/destination nodes through the virtual transponder node and the two virtual links as illustrated in Fig. 2 b.

Once the virtual graph is created, a set of possible paths from the virtual source to the virtual destination is computed based on the k-shortest path algorithm that returns all the paths within one hop from the shortest path. Paths are ordered from the least to the most congested in terms of available slots satisfying the request. The ordering procedure is based on the real path spectrum map obtained by intersecting the spectrum maps of all the links composing the path excluding the virtual links. In case of equally congested paths (those paths having the same real links but different virtual links), the ordering procedure follows the most to the least occupied transponder in order to avoid unused transponders when it is not necessary.

Once the paths are ordered, the algorithm selects a frequency slot applying the first-fit allocation schemes to the real path spectrum map and checks whether it is compatible with the virtual path spectrum map (the one including the virtual links). If it is not compatible, the algorithm moves to the next path and repeats the steps. If none of the selected slots matches the transponders, another loop through the paths is performed. This time the first-fit allocation is applied directly to the virtual spectrum map. The two loops foster the first-fit allocation on the real spectrum map trying to prevent high level of fragmentation. Finally, if no match is found, the lightpath request is blocked.

The proposed MWA-RSA scheme needs to know the state of the transponders of any node in the network. Since we consider to implement the proposed scheme in an EON using a GMPLS/PCE control plane, the transponder state information should be advertised by an extended version of the OSPF-TE protocol.

\section{Simulation scenario and results}

Simulations are performed using OPNET Modeler [6]. The developed model includes an implementation of the RSVPTE, PCEP and OSPF-TE protocols with the extensions required for EONs supporting different transponder architectures. A centralized GMPLS/PCE scenario is considered where the PCE stores a Traffic Engineering Database (TED) including network topology and the spectrum availability information.

The considered test network has a Spanish topology, with 30 nodes and 56 bidirectional links with 256 frequency slices per direction. The traffic is uniformly distributed among node pairs and LSPs arrive following a Poisson process, the mean holding time is fixed to 1 hour. The considered transponders are capable of generating four carriers, thus each transponder is equipped with four modulators and one laser (MW-SBVT architecture) or four lasers (ML-SBVT architecture). In case MW-SBVT architecture the maximum spacing allowed between carriers is $50 \mathrm{GHz}$ [3]. Two types of lightpath requests are considered: $100 \mathrm{Gbps}$ capacity lightpaths use 1 carrier occupying 3 slices $(37.5 \mathrm{GHz})$; $400 \mathrm{Gbps}$ capacity lightpaths use 4 carriers occupying 9 slices $(112.5 \mathrm{GHz})$ in case of ML-SBVT or 8 slices $(100$ $\mathrm{GHz}$ ) in case MW source (thanks to spectrally locked carriers) [4]. The two SBVT architectures are compared in terms of blocking probability $(\mathrm{Pb}) . \mathrm{Pb}$ is defined as the ratio between the blocked capacity and the overall requested capacity.

The proposed algorithm is compared with a multi-wavelength unaware RSA scheme (MWU-RSA) that does not consider the MW-SBVT constraints. When the MWU-RSA is applied, the PCE computes the set of shortest paths as in the MWA-RSA case; then the least congested path is selected and a first-fit spectrum assignment is performed on the path. Finally, the selected path and spectrum slot are returned to the ingress node that tries to allocate them from the most to the least used transponder, if partially allocated SBVT cannot be used, a new SBVT is activated. If a suitable transponder is found, the RSVP-TE signaling starts until it reaches the egress node, which does the same check between spectrum slot and available transponders. In case either the ingress or the egress node cannot find a suitable transponder to allocate the slot, the lightpath request is blocked.

Two network scenarios have been simulated: in the $400 G$ scenario only 400 Gbps lightpath requests are considered, in the Mix scenario the network load is equally distributed among $100 \mathrm{Gbps}$ and $400 \mathrm{Gbps}$ requests. Both scenarios consider a total simulation time of 500 days for each simulated point.

Fig. 3 shows $\mathrm{Pb}$ as a function of the number of transponders per node under the $400 \mathrm{G}$ scenario with fixed network 


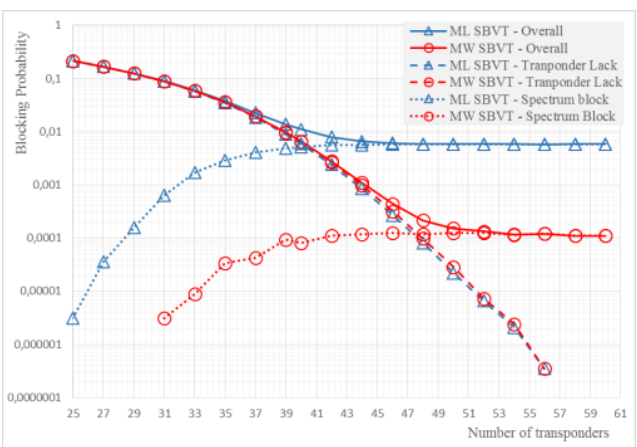

Fig. 3. Blocking probability vs transponders, $400 \mathrm{G}$ scenario.

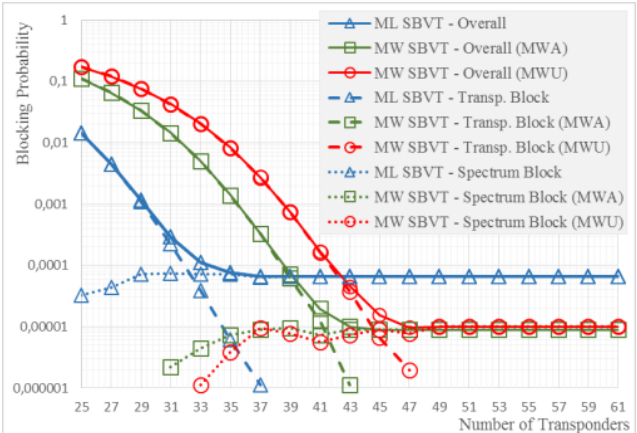

Fig. 5. Blocking probability vs transponders, Mix scenario

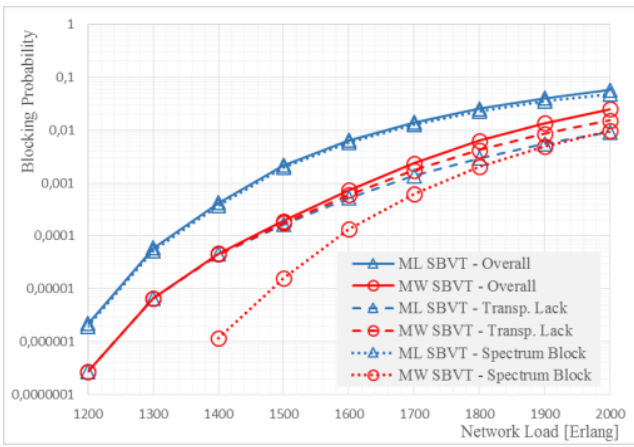

Fig. 4. Blocking probability vs load, $400 \mathrm{G}$ scenario.

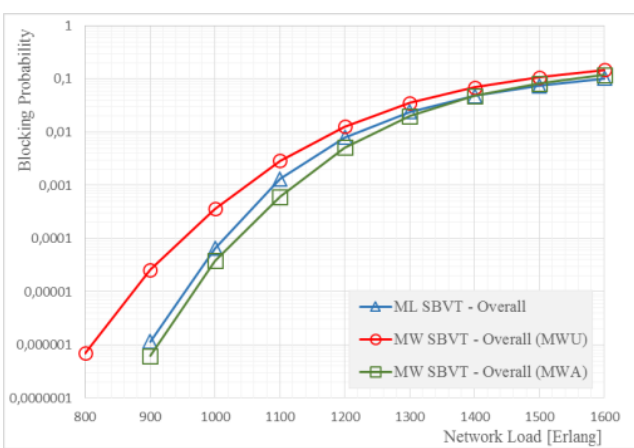

Fig. 6. Blocking probability vs load, Mix scenario.

load of 1600 Erlang. The transponder blocking component represents requests blocked due to lack of transponders, while the spectrum blocking component represents requests blocked due to spectrum unavailability (i.e., continuity constraint, contiguity constraint, spectrum lack). Results show that the spectrum compression introduced by the multiwavelength (i.e., 8 slices instead of 9) can bring important benefits to the network, in particular when the number of transponders is sufficient to sustain the network load. Indeed, the blocking probability is reduced more than one order of magnitude using a MW-SBVT. Fig. 4 shows $\mathrm{Pb}$ as a function of the network load in the $400 \mathrm{G}$ scenario with 45 transponders per node. The figure shows that achieved improvements are valid for a wide range of loads.

Fig. 5 shows $\mathrm{Pb}$ as function of number of transponders per node when the Mix scenario with fixed network load of 1000 Erlang. Results show that when few transponders are installed, the ML-SBVT performs better than the MWSBVT mainly because carriers can be allocated independently from the transponder thus $100 \mathrm{Gbps}$ lightpaths could be better allocated to partially used transponders leaving higher number of free transponders to accommodate 400 Gbps lightpaths. Similar to the $400 \mathrm{G}$ scenario, when the number of transponder increases, the transponder blocking component becomes marginal with respect to the spectrum blocking on behalf of the MW-SBVT. Comparing the two algorithms, we can see that the MWA-RSA achieves better results with respect to MWU-RSA. In particular, MWARSA allows to reduce the transponder blocking probability getting closer to the ML-SBVT blocking curve. Fig. 6 shows $\mathrm{Pb}$ as a function of the network load under the Mix scenario with 40 transponders per node. Results show that varying the load, the MWA-RSA still provides benefits, in particular in this case the blocking probability is lower with respect to the ML-SBVT.

\section{Conclusion}

This paper proposed a routing and spectrum assignment specifically designed for EONs equipped with S-BVT based on multi-wavelength (MW). Simulation results showed the scheme effectiveness in terms of blocking probability. Results also showed that MW is expected to be a valuable solution especially in the presence of super-channels, reducing blocking by more than one order of magnitude with respect to multi laser because of guard band reduction.

Acknowledgements: This research has been partially supported by the FP7 IDEALIST project.

\section{References}

[1] M. Jinno et al., "Multiflow optical transponder for efficient multilayer optical networking," Comm. Mag. SO, 2012.

[2] N. Sambo et al., "Sliceable transponder architecture including multiwavelength source," JOCN , vol.6, no.7, 590-600 (2014).

[3] N. Sambo et al., "First Demonstration of SDN-controlled SBVT based on Multi-wavelength Source with Programmable and Asymmetric Channel Spacing," in "Proc. of ECOC," (2014).

[4] M. Dallaglio et al., "Impact of SBVTs based on Multi-wavelength Source During Provisioning and Restoration in Elastic Optical Networks," in "Proc. of ECOC," (2014).

[5] "Opnet technologies," http://www.opnet.com. 